Int. J. Morphol.,

35(1):105-113, 2017

\title{
Morfología de la Placenta en Recién Nacidos con Defecto Congénito
}

\author{
Morphology of the Placenta in Newborns with Congenital Defects.
}

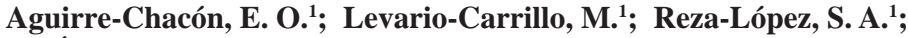
Ávila-Ochoa, R. ${ }^{2}$; Fierro-Murga, R. ${ }^{1}$ \& Chávez-Corral, D. V. ${ }^{1}$

AGUIRRE-CHACÓN, E. O.; LEVARIO-CARRILLO, M.; REZA-LÓPEZ, S. A.; ÁVILA-OCHOA, R.; FIERRO-MURGA, R. \& CHÁVEZ-CORRAL, D. V. Morfología de la placenta en recién nacidos con defecto congénito. Int. J. Morphol., 35(1):105-113, 2017.

RESUMEN: El objetivo del estudio fue comparar el índice de madurez placentaria y las alteraciones histopatológicas en placentas de recién nacidos/óbitos con y sin defecto congénito (DC). Se realizó un estudio tipo casos y controles. Se incluyeron $\mathrm{n}=25$ casos y $\mathrm{n}=50$ controles sin DC. Los casos se clasificaron de acuerdo a la presencia de DC aislado $(n=17)$ o múltiple $(n=8)$. Se incluyeron recién nacidos/óbitos $(\mathrm{RN})$ con una edad gestacional $(\mathrm{EG})>20$ semanas. Se excluyeron embarazos gemelares. Se recolectó información sobre las características clínicas del producto y de la madre, antropometría del RN y su evaluación de APGAR. Se examinó la placenta, macroscópica y microscópicamente, para determinar la presencia y extensión de alteraciones histopatológicas. Se determinó el índice de madurez placentaria (IMP), calculado dividiendo el número de membranas vásculo sinciciales en $1 \mathrm{~mm}^{2}$ entre el grosor de las mismas ( $\mu$ ). El IMP (media \pm DE) fue de $27.77 \pm 14$ en el grupo de controles, $30.31 \pm 12$ en el grupo de casos aislados y $16.76 \pm 1$ en el grupo de DC múltiple ( $\mathrm{p}<0.05)$. El resto de las variables evaluadas no mostraron diferencias significativas entre grupos. En conclusión este trabajo muestra una menor madurez placentaria asociada con la presencia de DC múltiple.

PALABRAS CLAVE: Defecto congénito; Madurez placentaria; Placenta; Alteraciones placentarias histopatológicas.

\section{INTRODUCCIÓN}

Los defectos congénitos se definen como aquellas anomalías estructurales o funcionales, que ocurren durante la vida intrauterina y se diagnostican durante el embarazo, en el parto o en un momento posterior de la vida (Aviña \& Tastekin, 2008).

La Organización Mundial de la Salud calcula que uno de cada 33 lactantes padece de algún tipo de DC, generando en 3.2 millones de personas algún grado de discapacidad cada año (OMS, 2015). En México, durante los años 2009 2010, $30.491(0.73 \%)$, de un total de 4, 123.531 nacimientos, cursaron con algún DC (Navarrete Hernández et al., 2013).

En la evaluación de los DC se sugiere un estudio integral de la madre, del recién nacido y de la placenta. Las alteraciones en la placenta con frecuencia se han asociado a co-morbilidad en el recién nacido (RN), ya que en ella se refleja lo acontecido durante la gestación (Stoll et al., 2003). Sin embargo, la evaluación de la placenta no se realiza de manera rutinaria (Weida et al., 2015). Se ha documentado que las placentas de recién nacidos (RNs) afectados por algún tipo de DC tienen en promedio un menor peso (Stoll et al.; Jones et al., 2015) comparado con placentas de RNs sin DC. Esta diferencia de peso es mayor en DC cardiacos (Jones et al.), múltiples o asociado a DC cromosómicos (Stoll et al.). Así mismo, se ha observado que en RNs afectados por DC cardiacos (Jones et al.), renales y del tubo neural (Castejón et al., 2006), la placenta no se desarrolla adecuadamente y es incapaz de proporcionar un ambiente apropiado para el desarrollo y crecimiento del feto.

Diversos estudios han informado que en embarazos complicados DC del tubo neural, cardiacos, cromosómicos y osteomusculares hay un incremento en la frecuencia en las lesiones de la placenta, asociadas a hipoxia como: mayor cantidad de depósitos de fibrina, fibrosis estromal, calcificaciones, daño en la pared de los vasos, trombosis intervellosa y presencia de células inflamatorias (Castejón S. et al., 2009; López et al., 2013 \& Jones et al.).

${ }^{1}$ Laboratorio de Embriología, Facultad de Medicina y Ciencias Biomédicas, Universidad Autónoma de Chihuahua, México.

${ }^{2}$ Hospital de Ginecoobstreticia No 15 del Instituto Mexicano del Seguro Social en el estado de Chihuahua, México. 
El desarrollo inadecuado de las vellosidades placentarias impide que la placenta responda correctamente a los requerimientos que necesita el feto para su desarrollo. En condiciones normales las vellosidades placentarias tienen un crecimiento lineal y van cambiando de ser inmaduras (brote trofoblástico, brote velloso y vellosidades intermedias) a maduras durante el tercer trimestre del desarrollo fetal (vellosidad intermedia madura, troncal y terminal) (Gratacós et al., 2007). Para evaluar la madurez de la placenta se han utilizado diversos indicadores como: el puntaje de Kaufmann y Bernischke sobre la maduración normal del las vellosidades placentarias (Benirschke \& Kaufmann, 2000), el porcentaje de nodos sinciciales (Jones \& Fox, 1977), el índice ecográfico de Grannum (Grannum, 1983), y el índice de madurez placentaria (IMP). Este último se basa en identificar el promedio de vellosidades terciarias en un $1 \mathrm{~mm}^{2}$, determinar el número de membranas vásculo sinciciales (MVS) y dividirlas entre su diámetro (Janowiak \& Mazerski, 1992).

La madurez placentaria se incrementa conforme avanza la edad gestacional. Sin embargo, se han observado cambios en este índice en diversas condiciones patológicas de la madre y del feto. En embarazos complicados por preeclampsia se ha observado un IMP mayor comparado con placentas de embarazos sin complicaciones hipertensivas (Ruiz-Quiñonez et al., 2014). El estado de salud del recién nacido (RN) es otro factor que puede modificar la madurez de la placenta (López et al.; Castejón et al.; Jones et al.). Se ha informado inmadurez placentaria en casos de DC cardiacos, del sistema osteomuscular y del tubo neural; caso contrario ocurre en los defectos que afectan al sistema renal, en los que se ha reportado hipermadurez placentaria (Castejón et al.). Para determinar esta condición se emplearon diversos métodos entre los que se incluyen cálculo de IMP, conteo de vellosidades maduras y terminales, así como el promedio de vasos sanguíneos por vellosidad (RuizQuiñonez et al.; Jones et al.; Castejón et al.).

Son escasos los estudios que han evaluado la morfología de la placenta en casos diagnosticados con defecto congénito de manera general; la información proviene de reporte de casos o bien de estudios que han evaluado defectos congénitos específicos (Castejón et al.; López et al.; Jones et al.). La característica más estudiada ha sido el peso de la placenta, el cual se ve más afectado en los casos de DC múltiple, de acuerdo a un estudio que incluyó un gran número de pacientes (Stoll et al.). Es posible que además del peso, la madurez de la placenta sea distinta cuando se trata de un DC aislado que cuando se observa un DC múltiple. Los objetivos de este estudio fueron: 1) comparar el índice de madurez placentaria en embarazos con y sin defectos congénitos y 2) determinar las alteraciones histopatológicas de placentas en estas condiciones.

\section{MATERIAL Y MÉTODO}

Se realizó un estudio de casos y controles. Se incluyeron pares madre/RN con edad gestacional mayor a 20 semanas, que aceptaron participar en el estudio. Los RN de embarazos múltiples fueron excluidos. Se evaluaron $n=25$ casos de $\mathrm{RN}$ con defecto congénito, de los cuales $\mathrm{n}=4$ óbitos y $\mathrm{n}=21$ nacidos vivos.

En el grupo control, se incluyeron dos RN sin DC por cada caso. Los casos de DC se clasificaron en dos grupos: con DC aislado $(n=17)$ y con DC múltiple $(n=8)$. Las muestras de placenta y los datos se recolectaron en los hospitales: Central del Estado y de Ginecología y Obstetricia número 15 del Instituto Mexicano del Seguro Social en la ciudad de Chihuahua, México.

Mujeres que acudieron a los hospitales antes mencionados internadas en el área de toco-cirugía fueron invitadas a participar en el estudio y se solicitó su consentimiento informado. Posteriormente, se realizó una entrevista donde se obtuvieron datos sobre antecedentes gineco-obstétricos, toxicomanías y antecedentes personales patológicos. La antropometría del RN fue evaluada por personal de enfermería previamente estandarizado: peso $(\mathrm{g})$, longitud $(\mathrm{cm})$, circunferencia cefálica $(\mathrm{cm})$, circunferencia torácica $(\mathrm{cm})$, circunferencia abdominal $(\mathrm{cm})$; y el APGAR fue evaluado (al minuto 1 y 5) por el pediatra o por el médico que atendió el evento obstétrico.

Los defectos congénitos se clasificaron según en el aparato y/o sistema que se afectó, de acuerdo a la Clasificación Internacional de Enfermedades CIE-10 y acorde al número de éstos, también se clasificaron como defectos aislados y múltiples. Se consideró defecto aislado aquél que afecta solamente una parte del cuerpo y como defectos múltiples cuando se observó un conjunto o patrón de anomalías relacionadas o no unas con otras (Romero Campos et al., 2010).

Análisis de tejido placentario. La recolección de la placenta se efectuó posterior al alumbramiento. En el examen macroscópico se midió su diámetro mayor y menor, se pesó y se revisó en busca de infartos, depósitos de fibrina, quistes y calcificaciones. Posteriormente se tomó una muestra de la cara materna en el área central la cual se fijó con formol al 3,7 \% y de la cual se obtuvieron laminillas teñidas con hematoxilina y eosina.

Índice de madurez placentaria. Con microscopio óptico (Axioscope 2 Plus, Carl Zeiss, Jena, Alemania), y el software AxioVision (AxioVision LE, v.4.6.2.0 Carl Zeiss MicroImaging, Alemania) se determinó el número de 
vellosidades placentarias terminales (rango entre $30-60 \mu$ ) encontradas en $1 \mathrm{~mm}^{2}$ y se calculó el promedio de lo encontrado en 5 campos a un aumento de 5x. En microfotografías a un aumento de 40x se midieron las siguientes estructuras: el diámetro mayor de la vellosidad terminal, número de MVS (porción más delgada de la membrana de la vellosidad libre de núcleos y nodos sinciciales), grosor de MVS (medido en la porción más delgada de la membrana) y el número de capilares por vellosidad terminal. Se calculó el promedio del grosor de la MVS en 10 vellosidades terminales. El IMP se obtuvo al dividir el número de MVS entre el grosor de las mismas $(\mu)$ (Janowiak \& Mazerski).

Análisis histopatológico. El estudio histopatológico fue realizado por un patólogo experto en identificar lesiones placentarias y su magnitud (calcificaciones, hemorragias, edema vellositario, fibrosis, trombosis intervellosa, quistes, infarto reciente e infarto antiguo). Estas lesiones se calificaron de acuerdo a lo sugerido por Loardi et al. (2016) en una escala semi-cuantitativa donde: 0 , es ausencia de lesión; 1 , lesión en 1 a 5 campos; 2, lesión en 6 a 10 campos; 3, lesión en más de 10 campos.

Análisis estadístico. Los datos se presentan como media \pm D.E., o como frecuencias y porcentajes, de acuerdo a la escala de medición de las variables. Los grupos se compararon utilizando las pruebas de $\mathrm{chi}^{2}$ o exacta de Fisher en el caso de variables medidas en escala nominal u ordinal. Para las variables medidas en escala de razón se utilizó la prueba de t de Student, análisis de varianza o prueba de Kruskal Wallis de acuerdo a la distribución de las variables. Las características relacionadas con la evaluación de la madurez placentaria (número de vellosidades terminales, número de MVS, grosor de la MVS y el IMP) fueron ajustadas por edad gestacional, género del recién nacido, peso al nacer del producto, óbito o RN vivo, presencia de

Tabla I. Clasificación de defectos congénitos según (CIE-10).

\begin{tabular}{lll}
\hline Tipo de defecto & $\mathbf{n}$ & $\mathbf{\%}$ \\
\hline Sistema nervioso & 4 & 12 \\
Sistema circulatorio & 6 & 17 \\
Fisura del paladar y labio hendido & 2 & 6 \\
Otras del sistema digestivo & 5 & 15 \\
Órganos genitales & 3 & 9 \\
Sistema urinario & 7 & 20 \\
Sistema osteomuscular & 4 & 12 \\
Otras malformaciones congénitas & 2 & 6 \\
Cromosómicas & 1 & 3 \\
\hline Total & 34 & 100 \\
\hline
\end{tabular}

*El total de defectos congénitos es mayor al número de pacientes ya que 8 casos presentaron defectos múltiples en los siguientes aparatos y sistemas: Circulatorio, digestivo, paladar, osteomuscular, renal y nervioso. complicaciones hipertensivas, diabetes, peso placentario y número de gesta) mediante un modelo de regresión múltiple. Se consideró una diferencia significativa cuando el valor de p fue <0,05. El análisis se realizó utilizando el software STATA 11.0 (Stata Statistical software. Release 11.0 College Station, Tx:stata).

\section{RESULTADOS}

Los principales defectos congénitos (incluyendo defectos múltiples) identificados afectaron a los sistemas: urinario, circulatorio, nervioso y digestivo (Tabla I).

La presencia de alguna co-morbilidad al momento del nacimiento (insuficiencia respiratoria, bajo peso al nacer, restricción del crecimiento y prematurez) fue mayor en el grupo de pacientes con defecto congénito (48\%) comparado con los controles $(24 \%)$ [p=0,03]. (Tabla II).

La antropometría del $\mathrm{RN}$, el peso y diámetros de la placenta no mostraron diferencias significativas en los tres grupos como se puede observar en la Tabla III.

La Tabla IV muestra la morfología de las vellosidades placentarias, se observó un menor número de vellosidades terminales y de MVS en las placentas con DC múltiple lo que condicionó un IMP menor en este grupo como se muestra en la Figura 1.

No se identificó diferencia en las alteraciones histopatológicas en los grupos estudiados en cuanto a la ocurrencia de las alteraciones histopatológicas o a la extensión de las mismas (Tablas V y VI)

\section{DISCUSIÓN}

Nuestro estudio muestra que las placentas de embarazos complicados con DC múltiples presentaron un menor índice de madurez placentaria. Sin embargo, no se observaron diferencias en la frecuencia de alteraciones histopatológicas o en las características macroscópicas de la placenta.

En la Tabla VII se resumen los estudios que han evaluado placentas de $\mathrm{RN}$ con defecto congénito y su relación con la madurez placentaria. Diversos autores (Jones et al.; López et al.; Castejón et al.) han informado resultados similares a los observados en este estudio, en casos de embarazos complicados en DC. 
Tabla II. Características clínicas de la población estudiada.

\begin{tabular}{|c|c|c|c|}
\hline Variable & $\begin{array}{c}\text { Sin defecto } \\
\mathbf{n}=\mathbf{5 0} \\
\mathbf{X} \pm \mathbf{D E} / \mathbf{n}(\%)\end{array}$ & $\begin{array}{c}\text { Con defecto } \\
\text { n=25 } \\
X \pm \text { DE } / \mathbf{n}(\%)\end{array}$ & $\mathbf{p}$ \\
\hline Edad materna (años) & $25 \pm 7$ & $27 \pm 6$ & 0,26 \\
\hline \multicolumn{4}{|l|}{ Escolaridad } \\
\hline Secundaria o menor & $28(56)$ & $10(40)$ & \multirow[t]{2}{*}{0,19} \\
\hline Preparatoria o mayor & $22(44)$ & $15(60)$ & \\
\hline \multicolumn{4}{|l|}{ Ocupación } \\
\hline Dentro del hogar & $24(52)$ & $12(48)$ & \multirow[t]{2}{*}{0,74} \\
\hline Fuera del hogar & $22(48)$ & $13(52)$ & \\
\hline Peso pregestacional (kg) & $69 \pm 22$ & $61 \pm 10$ & 0,33 \\
\hline Talla materna $(\mathrm{cm})$ & $160 \pm 5$ & $162 \pm 6$ & 0,41 \\
\hline $\begin{array}{l}\text { Índice de masa corporal } \\
\text { pregestacional }\left(\mathrm{kg} / \mathrm{m}^{2}\right)\end{array}$ & $26 \pm 8$ & $23 \pm 3$ & 0,13 \\
\hline \multicolumn{4}{|l|}{ Sobrepeso pregestacional* } \\
\hline $\mathrm{Si}$ & $13(55)$ & $9(25)$ & \multirow[t]{2}{*}{0,23} \\
\hline No & $16(45)$ & $3(25)$ & \\
\hline $\begin{array}{l}\text { Ganancia de peso durante el } \\
\text { embarazo }(\mathrm{kg})\end{array}$ & $10 \pm 7$ & $12 \pm 3$ & 0,09 \\
\hline \multicolumn{4}{|l|}{ Tabaquismo } \\
\hline Sí & $5(10)$ & $0(0)$ & \multirow[t]{2}{*}{0,11} \\
\hline No & $45(90)$ & $25(100)$ & \\
\hline \multicolumn{4}{|l|}{ Alcoholismo } \\
\hline Sí & $3(6)$ & $2(8)$ & \multirow[t]{2}{*}{1,00} \\
\hline No & $47(94)$ & $23(92)$ & \\
\hline
\end{tabular}

*Este valor se obtuvo con 48 observaciones, se definió como obesidad un IMC mayor o igual a 25,0 $\mathrm{kg} / \mathrm{m}^{2}$

Tabla III. APGAR, características macroscópicas de la placenta y antropometría al nacer.

\begin{tabular}{lcccc}
\hline Variable & Sin defecto & \multicolumn{2}{c}{ Con defecto } & p \\
& & Aislado & Múltiple \\
& $\mathrm{n}=50$ & $\mathrm{n}=17$ & $\mathrm{n}=8$ \\
& $\mathrm{X} \pm \mathrm{DE} / \mathrm{n}(\%)$ & $\mathrm{X} \pm \mathrm{DE} / \mathrm{n}(\%)$ & $\mathrm{X} \pm \mathrm{DE} / \mathrm{n}(\%)$ \\
\hline APGAR (1 minuto) & $8 \pm 1$ & $7 \pm 2$ & $5 \pm 4$ & 0,08 \\
APGAR (5 minutos) & $9 \pm 0$ & $8 \pm 2$ & $5 \pm 4$ & 0,13 \\
Sexo & & & \\
$\quad$ Masculino & $28(56)$ & $7(41)$ & $6(75)$ & 0,30 \\
Femenino & $22(44)$ & $10(59)$ & $2(25)$ & \\
Peso (g) & $2542 \pm 899$ & $2690 \pm 1033$ & $2746 \pm 794$ & 0,60 \\
Longitud (cm) & $49 \pm 7$ & $49 \pm 6$ & $49 \pm 4$ & 0,76 \\
Circunferencia cefálica (cm) & $32 \pm 4$ & $33 \pm 5$ & $34 \pm 2$ & 0,37 \\
Circunferencia torácica (cm) & $30 \pm 4$ & $32 \pm 6$ & $31 \pm 4$ & 0,46 \\
Circunferencia abdominal (cm) & $29 \pm 5$ & $33 \pm 6$ & $29 \pm 5$ & 0,07 \\
Peso de la placenta (g) & $427 \pm 157$ & $443 \pm 214$ & $336 \pm 135$ & 0,56 \\
Diámetro mayor de la placenta (cm) & $17 \pm 3$ & $18 \pm 3$ & $18 \pm 2$ & 0,48 \\
Diámetro menor de la placenta $(\mathrm{cm})$ & $15 \pm 2$ & $15 \pm 3$ & $16 \pm 2$ & 0,30 \\
Edad gestacional (semanas) & $35 \pm 4$ & $35 \pm 5$ & $35 \pm 4$ & 0,69 \\
Prematurez & & & & 0,97 \\
$\quad$ Sí & $27(54)$ & $9(52)$ & $4(50)$ & \\
$\quad$ No & $23(46)$ & $8(47)$ & $4(50)$ & \\
\hline
\end{tabular}

*Diámetro menor no tuvo distribución normal por lo que se uso la prueba de Kwallis. 
Tabla IV. Morfología de vellosidades e índice de madurez placentaria (IMP)

\begin{tabular}{|c|c|c|c|}
\hline \multirow[t]{4}{*}{ Variable } & \multirow{4}{*}{$\begin{array}{c}\text { Sin defecto } \\
\begin{array}{c}n=50 \\
X \pm D E\end{array}\end{array}$} & \multicolumn{2}{|c|}{ Con defecto } \\
\hline & & Aislado & Múltiple \\
\hline & & $\mathrm{n}=17$ & $\mathrm{n}=8$ \\
\hline & & $\mathrm{X} \pm \mathrm{DE}$ & $\mathrm{X} \pm \mathrm{DE}$ \\
\hline Número de vellosidades terminales $\left(1 \mathrm{~mm}^{2}\right)$ & $19 \pm 7^{\mathrm{a}}$ & $20 \pm 8 \mathrm{a}$ & $13 \pm 6^{\mathrm{b}}$ \\
\hline Número de MVS* en $1 \mathrm{~mm}^{2}$ & $27 \pm 12 \mathrm{a}$ & $30 \pm 10^{\mathrm{a}}$ & $18 \pm 10 \mathrm{~b}$ \\
\hline Grosor de MVS $(\mu)$ & $1,11 \pm 0,4$ & $1,13 \pm 0,4$ & $1,30 \pm 0,4$ \\
\hline Índice de madurez placentaria & $27,77 \pm 14 \mathrm{a}$ & $30,31 \pm 12^{\mathrm{a}}$ & $16,76 \pm 1^{b}$ \\
\hline
\end{tabular}

*MVS= membrana vásculo sincicial. El IMP se calcula al dividir el número de MVS en $1 \mathrm{~mm}^{2}$ entre su grosor. Diferencias significativas ( $\mathrm{p}<0.05$ ) fueron observadas en los grupos, señalados con distinto superíndice. Después de ajustar por edad gestacional, genero del recién nacido, peso al nacer del producto, óbito o paciente vivo, enfermedad gestacional hipertensiva, diabetes, peso placentario y número de gesta.
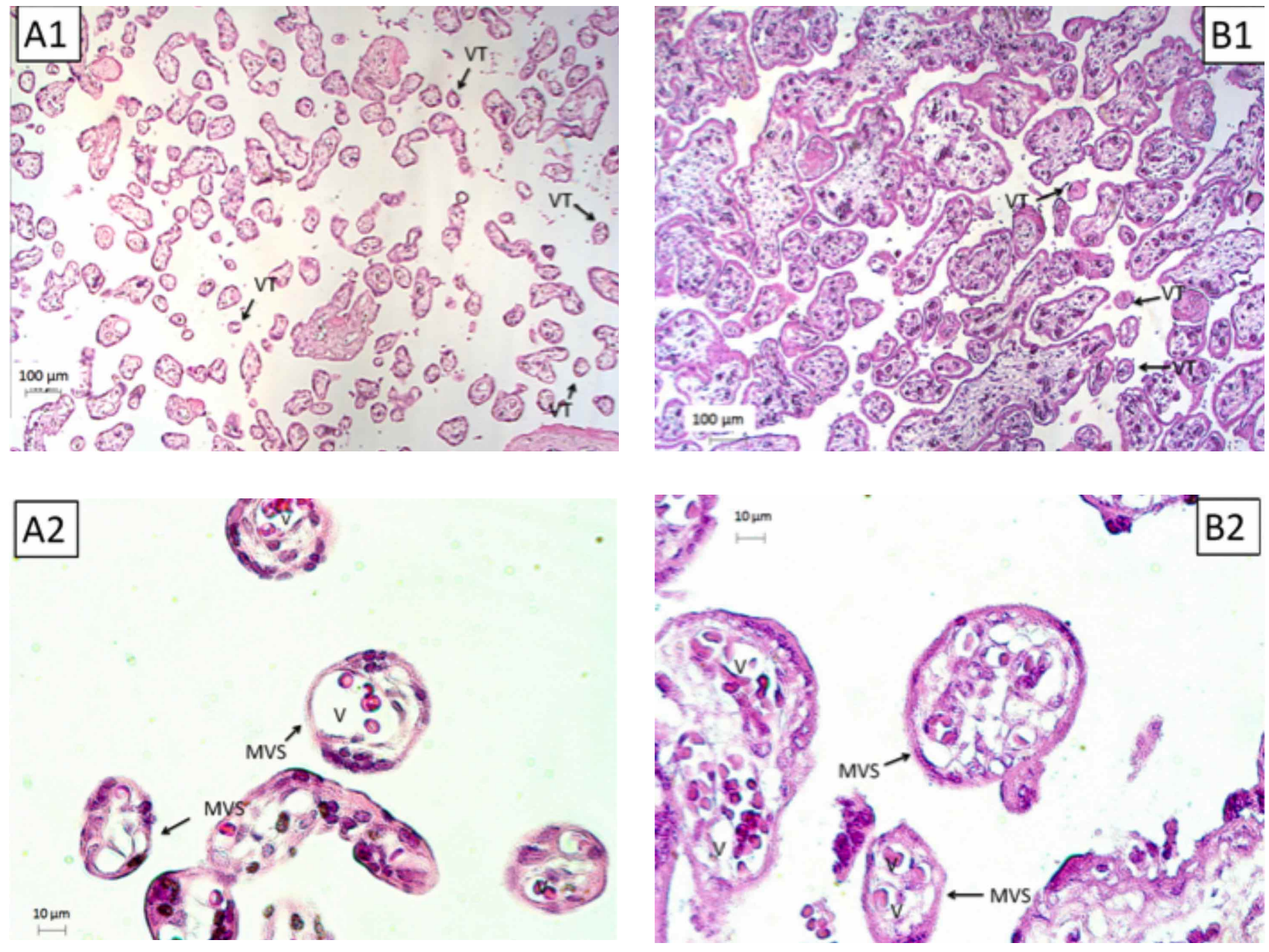

Fig. 1. Microfotografías de área central de placentas Pre término (33 semanas). Grupos de estudio: (A1) control sin defecto Congénito aumento 5x; (A2) control sin defecto congénito aumento 40x, (B1) caso con múltiples defectos congénitos (sistema renal y digestivo), aumento 5x; y (B2) caso con múltiples defectos congénitos (sistema renal y digestivo) aumento 40x; El número de vellosidades terminales y MVS es más abundante en el grupo de controles por lo que su IMP es mayor si se compara con el grupo de defectos múltiples. MVS= Membranas vásculo sinciciales. VT= Vellosidad terminal. V= Vaso sanguíneo. 
Tabla V. Alteraciones histopatológicas de la placenta.

\begin{tabular}{|c|c|c|c|c|}
\hline \multirow[t]{2}{*}{ Variable } & \multirow{2}{*}{$\begin{array}{c}\text { Sin defecto } \\
\begin{array}{c}n=50 \\
n(\%)\end{array}\end{array}$} & \multicolumn{2}{|c|}{ Con defecto } & \multirow[t]{2}{*}{$\mathbf{p}$} \\
\hline & & $\begin{array}{c}\text { Aislado } \\
\mathrm{n}=17 \\
\mathrm{n}(\%)\end{array}$ & $\begin{array}{c}\text { Múltiple } \\
n=8 \\
n(\%)\end{array}$ & \\
\hline \multicolumn{5}{|l|}{ Calcificación } \\
\hline $\mathrm{Si}$ & $26(52)$ & $9(53)$ & $4(50)$ & 1,0 \\
\hline No & $24(48)$ & $8(47)$ & $4(50)$ & \\
\hline \multicolumn{5}{|l|}{ Hemorragia } \\
\hline $\mathrm{Si}$ & $10(20)$ & $3(18)$ & $0(0)$ & 0,56 \\
\hline No & $40(80)$ & $14(82)$ & $8(100)$ & \\
\hline \multicolumn{5}{|c|}{ Edema vellositario* } \\
\hline $\mathrm{Si}$ & $4(8)$ & $3(18)$ & $1(12)$ & \\
\hline No & $45(92)$ & $14(82)$ & $7(83)$ & 0,42 \\
\hline \multicolumn{5}{|c|}{ Fibrosis intervellosa } \\
\hline $\mathrm{Si}$ & $16(32)$ & $3(18)$ & $1(12)$ & \\
\hline No & $34(68)$ & $14(82)$ & $7(83)$ & 0,42 \\
\hline \multicolumn{5}{|c|}{ Trombosis intervellosa } \\
\hline $\mathrm{Si}$ & $48(96)$ & $17(100)$ & $8(100)$ & \\
\hline No & $2(4)$ & $0(0)$ & $0(0)$ & 1,0 \\
\hline \multicolumn{5}{|c|}{ Infarto reciente* } \\
\hline $\mathrm{Si}$ & $12(24)$ & $5(31)$ & $3(38)$ & \\
\hline No & $37(76)$ & $11(69)$ & $5(62)$ & 0,69 \\
\hline \multicolumn{5}{|c|}{ Infarto antiguo* } \\
\hline $\mathrm{Si}$ & $25(51)$ & $6(35)$ & $2(25)$ & \\
\hline No & $24(49)$ & $11(65)$ & $6(75)$ & 0,26 \\
\hline
\end{tabular}

*En algunos de los parámetros no fue posible evaluar el total de las muestras.

Una mayor frecuencia de inmadurez placentaria se ha reportado en otras condiciones como en placentas de RN prematuros con restricción del crecimiento fetal idiopática, malformaciones congénitas, anomalías cromosómicas o genéticas y de madres con diagnóstico de diabetes mellitus (Redline, 2012). Se ha sugerido que la inmadurez en las placentas de productos con DC múltiples pudiera ocurrir como un mecanismo de respuesta adaptativa ante las condiciones fetales desfavorables [defectos del tubo neural, cardiacos, osteomusculares y cromosómicos] (López et al.; Jones et al.; Castejón et al.) o bien, como respuesta a procesos moleculares asociados al factor de crecimiento endotelial y al factor de crecimiento placentario (Jones et al.) o por morbilidad materna pre-existente (Ruiz-Quiñonez et al.; Huynh et al., 2015; Loardi et al.).

El estudio histopatológico rutinario de la placenta permite identificar cambios en la estructura de la vellosidad, así como de sus vasos y del espacio intervelloso, lo que proporciona información acerca de su desarrollo y del entorno donde se desarrolla el producto.
Las alteraciones reportadas no muestran diferencia entre los grupos estudiados. La trombosis intervellosa fue la alteración más observada en los tres grupos. En contraste, se han reportado el edema y la necrosis como las principales alteraciones histopatológicas de la placenta de $\mathrm{RN}$ con defectos del tubo neural. Estas lesiones se han asociado a mala perfusión fetal en casos de defecto del tubo neural (López et al.; Castejón S. et al.). El predominio de otras lesiones en general y la falta de diferencia entre grupos en nuestro estudio podrían sugerir que las condiciones varían acorde al tipo de defecto congénito o está influenciado por otros factores.

En cuanto al peso de la placenta, se ha reportado que es menor en productos con DC cardiaco (Cuna et al., 2014). En contraste, otros autores han encontrado un peso placentario más bajo en los controles que en placentas de RN con DC aislado (Stoll et al.). En nuestro estudio, el peso de las placentas fue ligeramente menor en casos de defecto congénito múltiple (336 g) pero no se determinó una diferencia significativa en comparación con los controles (427 g) y casos con DC aislado (443 g). 
Tabla VI. Extensión de las lesiones placentarias.

\begin{tabular}{|c|c|c|c|c|}
\hline \multirow[t]{2}{*}{ Variable } & \multirow{2}{*}{$\begin{array}{c}\text { Sin defecto } \\
\begin{array}{c}n=50 \\
n(\%)\end{array}\end{array}$} & \multicolumn{2}{|c|}{ Con defecto } & \multirow[t]{2}{*}{$\mathbf{p}$} \\
\hline & & $\begin{array}{c}\text { Aislado } \\
\mathrm{n}=17 \\
\mathrm{n}(\%)\end{array}$ & $\begin{array}{c}\text { Múltiple } \\
n=8 \\
n(\%)\end{array}$ & \\
\hline \multicolumn{5}{|l|}{ Grado calcificación } \\
\hline 0 & $24(48)$ & $8(50)$ & $5(63)$ & \\
\hline 1 & $22(44)$ & $6(38)$ & $2(25)$ & \\
\hline 3 & $4(8)$ & $2(12)$ & $1(12)$ & 0,19 \\
\hline \multicolumn{5}{|l|}{ Grado hemorrag ia } \\
\hline 0 & $40(82)$ & $13(81)$ & $8(100)$ & \\
\hline 1 & $5(10)$ & $1(6)$ & 0 & \\
\hline 2 & $2(4)$ & $2(13)$ & 0 & \\
\hline 3 & $2(4)$ & 0 & 0 & 0,82 \\
\hline \multicolumn{5}{|l|}{ Grado edema } \\
\hline 0 & $45(92)$ & $14(82)$ & $7(83)$ & \\
\hline 1 & $3(6)$ & $1(6)$ & $1(13)$ & \\
\hline 2 & 0 & $1(6)$ & 0 & \\
\hline 3 & $1(2)$ & $1(6)$ & 0 & 0,41 \\
\hline \multicolumn{5}{|l|}{ Grado fibrosis } \\
\hline 0 & $33(66)$ & $14(82)$ & $6(75)$ & \\
\hline 1 & $12(24)$ & $3(18)$ & $2(25)$ & \\
\hline 2 & $4(8)$ & 0 & 0 & \\
\hline 3 & $1(2)$ & 0 & 0 & 0,88 \\
\hline \multicolumn{5}{|l|}{ Grado trombosis } \\
\hline 0 & $3(6)$ & $11(69)$ & $5(71)$ & \\
\hline 1 & $21(44)$ & $1(6)$ & $2(29)$ & \\
\hline 2 & $10(21)$ & $1(6)$ & 0 & \\
\hline 3 & $14(25)$ & $3(19)$ & 0 & 0,14 \\
\hline \multicolumn{5}{|l|}{ Extensión infarto } \\
\hline 0 & $36(75)$ & $11(69)$ & $5(63)$ & \\
\hline 1 & $9(19)$ & $5(31)$ & $3(37)$ & \\
\hline 2 & $2(4)$ & 0 & 0 & \\
\hline 3 & $1(2)$ & 0 & 0 & \\
\hline Extensión infarto & & & & 0,74 \\
\hline 0 & $24(50)$ & $11(69)$ & $5(71)$ & \\
\hline 1 & $15(31)$ & $1(6)$ & $2(29)$ & \\
\hline 2 & $6(13)$ & $1(6)$ & 0 & \\
\hline 3 & $3(6)$ & $3(19)$ & 0 & 0,23 \\
\hline \multicolumn{5}{|l|}{ Extensión quistes } \\
\hline 0 & $43(86)$ & $17(100)$ & $7(88)$ & \\
\hline 1 & $5(10)$ & 0 & $1(12)$ & \\
\hline 2 & $2(4)$ & 0 & 0 & 0,58 \\
\hline
\end{tabular}

$\mathrm{O}=$ Sin lesiones, $1=$ Lesión en 1 a 5 campos, $2=$ Lesión en 6 a 10 campos y $3=$ Lesión en más de 10 campos. En algunos de los parámetros no fue posible evaluar el total de las muestras.

En este estudio se observó un mayor número de DC aislados y menor de DC múltiples. Los principales órganos y sistemas afectados fueron: el sistema urinario, nervioso, digestivo y circulatorio. Estos datos son semejantes a lo reportado por Egbe et al. (2014) al analizar la base de datos The National Inpatient Sample (NIS) de Estados Unidos de América y coinciden con lo reportado en la literatura nacional e internacional (Stoll et al.; Montes-Núñez et al., 2011; Navarrete Hernández et al.) excepto la hidronefrosis, la cual se encontró con mayor frecuencia de lo esperado. Factores como obesidad (Källén, 1998), tabaquismo y alcoholismo maternos (Pei et al., 2015) se han asociado con la ocurrencia de defectos congénitos. Sin embargo, en nuestro estudio dichas características no variaron entre los grupos. Otros factores relacionados con la frecuencia de DC tales como factores psicológicos maternos, exposición a insecticidas o solventes orgánicos, aplicación de vacunas o contacto con pacientes con enfermedades febriles (Bretón et al., 2012) no fueron evaluados en este estudio.

Entre las potenciales limitaciones se podría mencionar el tamaño de la muestra evaluada, lo cual no permitió examinar las lesiones histopatológicas de la placenta de acuerdo al tipo de DC. Otra limitante la constituye el hecho de no contar con información sobre cariotipo y no poder asociar defectos congénitos con defectos cromosómicos, en los cuales se ha observado un menor peso placentario (Stoll et al.). Dado la naturaleza transversal del estudio, no fue posible realizar un seguimiento de los RN para comprobar la gravedad del defecto congénito y descartar la presencia de otro tipo de DC diagnosticado en etapas posteriores de la vida.

En conclusión, un menor número de vellosidades terminales por $\mathrm{mm}^{2}$ y una menor cantidad de MVS y por ende un IMP menor se asoció con la presencia de defectos congénitos múltiples. Las alteraciones histopatológicas no mostraron diferencias entre placentas de $\mathrm{RN}$ con DC comparado con las de RN sin DC.

\section{AGRADECIMIENTOS}

Agradecemos la valiosa participación de los pacientes y a la Facultad de Medicina y Ciencias Biomédicas, Universidad Autónoma de Chihuahua, México por el apoyo brindado para la realización de este estudio. 


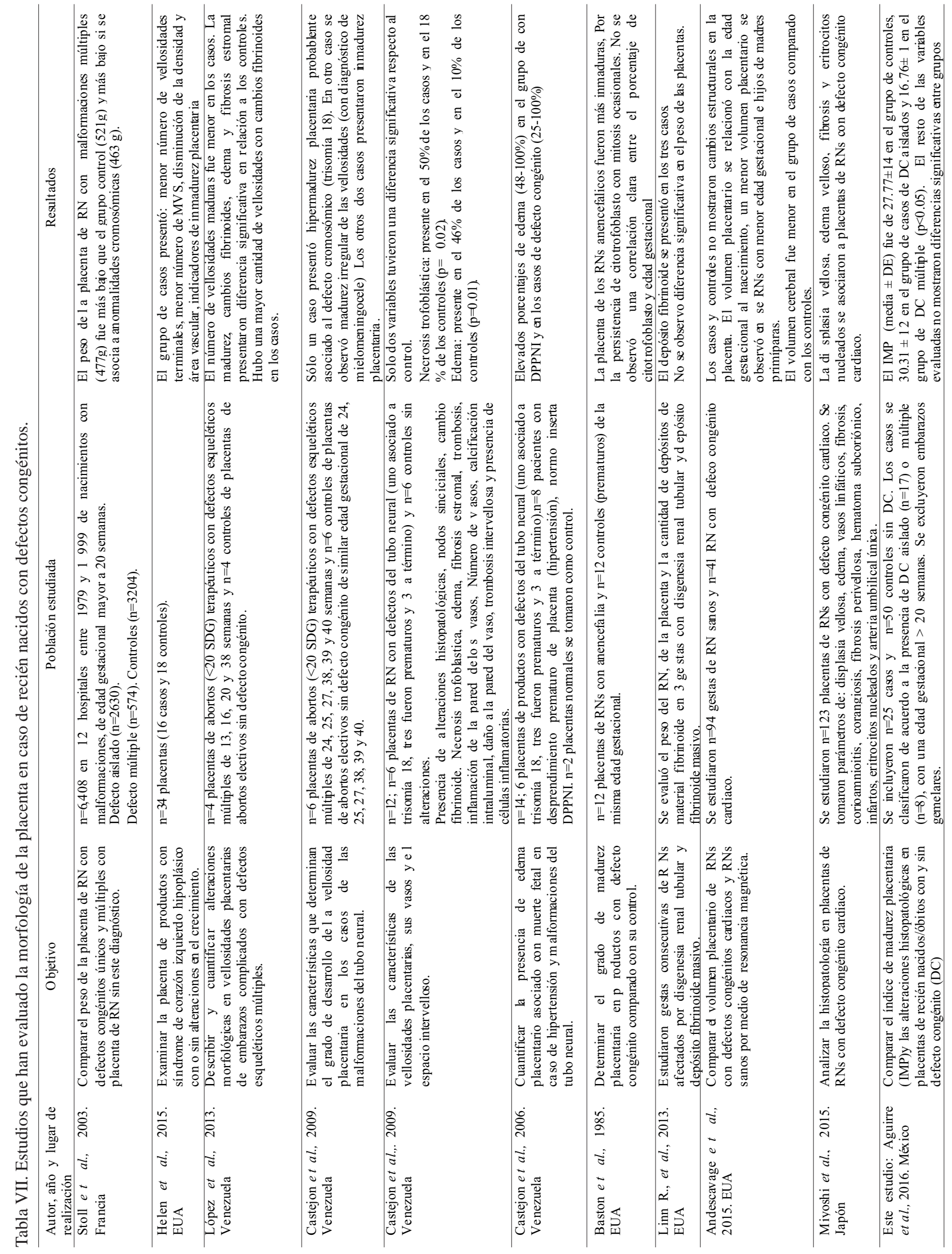


AGUIRRE-CHACóN, E. O.; LEVARIO-CARRILlO, M.; REZA-LÓPEZ, S. A.; ÁVILA-OCHOA, R.; FIERRO-MURGA, R.; CHÁVEZ-CORRAL, D. V. Morphology of the placenta in newborns with congenital defects. Int. J. Morphol., 35(1):105-113, 2017.

SUMMARY: The objective of the study was to compare the placental maturity index (PMI) and pathohistological alterations of placentas from newborn/stillborns (NB) with or without congenital defects (CD). A case control study was carried out. N=25 CD cases and $\mathrm{n}=50$ controls without $\mathrm{CD}$ were included. Cases were classified according to the presence of simple $(\mathrm{n}=17)$ or multiple $(\mathrm{n}=8) \mathrm{CD}$. Newborn/ stillborns with gestational age $>20$ weeks were included. Twin pregnancies were excluded. We collected information on clinical characteristics of the NB and the mother, NB anthropometry and APGAR score. The placenta was macro and microscopically examined to determine the presence and extension of histological alterations. The PMI was obtained by dividing the number of vasculo-syncytial membranes in $1 \mathrm{~mm}^{2}$, by their thickness $(\mu)$. The PMI (Mean \pm SD) was $27.77 \pm 14$ in the control group, $30.31 \pm 12$ in the group diagnosed with simple CD, and $16.76 \pm 1$ in the group diagnosed with multiple $\mathrm{CD}(\mathrm{p}<0.05$. The rest of the assessed variables did not show significant difference between groups. In conclusion, this work shows a lower placental maturity associated with the presence of multiple CD.

KEY WORDS: Congenital defects; Placental maturity; Placenta; Histological alterations of placenta.

\section{REFERENCIAS BIBLIOGRÁFICAS}

Aviña, F. J. A. \& Tastekin, A. Malformaciones congénitas: clasificación y bases morfogénicas. Rev. Mex. Pediatr., 75(2):71-4, 2008.

Benirschke, K. \& Kaufmann, P. Pathology of the Human Placenta. $4^{\text {th }}$ ed. New York, Springer, 2000.

Bretón, C. O.; Manrique, F. L.; Barajas, S. J. \& Velandia, J. A. Factores de riesgo materno asociados a cardiopatías congénitas en el nororiente colombiano. Pediatría, 45(2):108-23, 2012.

Castejón, O. C.; Luzardo, C.; Rivas, A. E.; Santiago, M.; Lugo, J. \& Sifontes, C. Madurez de las vellosidades coriales en relación con malformaciones del tubo neural durante el tercer trimestre del embarazo. Rev. Obstet. Ginecol. Venez., 66(4):241-50, 2006.

Castejón S., O. C.; López G., Á. J.; Castejón M., O. C.; Pérez-Ybarra, L. M.; Quiroz, D. \& Castejón S., O. A. Cambios degenerativos coriónicos asociados a malformaciones fetales del tubo neural durante el tercer trimestre del embarazo. Rev. Obstet. Ginecol. Venez., 69(1):20-7, 2009.

Cuna, A.; Hakima, L.; Tseng, Y. A.; Fornier, B.; Islam, S.; Quintos-Alagheband, M. L.; Khullar, P.; Weinberger, B. \& Hanna, N. Clinical dilemma of positive histologic chorioamnionitis in term newborn. Front. Pediatr., 2:27, 2014.

Egbe, A.; Lee, S.; Ho, D.; Uppu, S. \& Srivastava, S. Prevalence of congenital anomalies in newborns with congenital heart disease diagnosis. Ann. Pediatr. Cardiol., 7(2):86-91, 2014.

Grannum, P. A. Ultrasound examination of the placenta. Clin. Obstet. Gynaecol., 10(3):459-73, 1983.

Gratacós, E.; Gómez, R.; Nicolaides, K.; Romero Galue, R. \& Cabero Roura, L. Medicina Fetal. Madrid, Editorial Médica Panamericana, 2007.

Huynh, J.; Dawson, D.; Roberts, D. \& Bentley-Lewis, R. A systematic review of placental pathology in maternal diabetes mellitus. Placenta, 36(2):101$14,2015$.

Janowiak, Z. \& Mazerski, J. New criterion of placental barrier maturity. Zentralbl. Gynakol., 114(6):292-5, 1992.

Jones, C. J. \& Fox, H. Syncytial knots and intervillous bridges in the human placenta: an ultrastructural study. J. Anat., 124(Pt. 2):275-86, 1977.

Jones, H. N.; Olbrych, S. K.; Smith, K. L.; Cnota, J. F.; Habli, M.; RamosGonzales, O.; Owens, K. J.; Hinton, A. C.; Polzin, W. J.; Muglia, L. J. \& Hinton, R. B. Hypoplastic left heart syndrome is associated with structural and vascular placental abnormalities and leptin dysregulation. Placenta, 36(10):1078-86, 2015.

Källén, K. Maternal smoking, body mass index, and neural tube defects. Am. J. Epidemiol., 147(12):1103-11, 1998.

Loardi, C.; Falchetti, M.; Prefumo, F.; Facchetti, F. \& Frusca, T. Placental morphology in pregnancies associated with pregravid obesity. J. Matern. Fetal Neonatal Med., 29(16):2611-6, 2016.

López, Á.; Castejón, O.; Pérez, L.; Salazar, A.; Rodríguez, G. \& Urdaneta, J. Alteraciones morfológicas de las vellosidades placentarias asociadas a malformaciones fetales múltiples del sistema esquelético. Rev. Bras. Saude Mater. Infant., 13(3):207-14, 2013.

Montes-Núñez, S.; Chávez-Corral, D. V.; Reza-López, S.; Sanin, L. H.; AcostaMaldonado, B. \& Levario-Carrillo, M. Birth weight in children with birth defects. Birth Defects Res. A Clin. Mol. Teratol., 91(2):102-7, 2011.

Navarrete Hernández, E.; Canún Serrano, S.; Reyes Pablo, A. E.; Sierra Romero, M. del C. \& Valdés Hernández, J. Prevalencia de malformaciones congénitas registradas en el certificado de nacimiento y de muerte fetal: México, 2009-2010. Bol. Med. Hosp. Infant. Mex., 70(6):499-505, 2013.

Organización Mundial de la Salud (OMS). Anomalías Congénitas. Ginebra, Organización Mundial de la Salud, 2015. Available: http://www.who.int/ mediacentre/factsheets/fs370/es/

Pei, L.; Kang, Y.; Cheng, Y. \& Yan, H. The association of maternal lifestyle with birth defects in Shaanxi Province, Northwest China. PLoS One, 10(9):e0139452, 2015.

Redline, R. W. Distal villous immaturity. Diagn. Histopathol., 18(5):189-94, 2012.

Romero Campos, A.; Díaz Martinez, A.; Fornieles García, Y.; Calero Romero, L.; Irala Pérez, F. J.; López Torné, M. M.; Linares Navarro, M. J.; Ramos Vásquez, M. A.; Conejo Gómez, C.; Casado Fernández, E.; Ferreras Fernández, M. J.; Martínez Reina, A.; Pena González, J.; Delgado Alés, J.; Águila González, A \& Sánchez García, M. Codificación en CIE-9-MC Anomalías congénitas. En: Fornieles García, Y. \& Díaz Martínez, A. (Eds.). Codificación en CIE-9-MC Anomalías congénitas. Andalucía, Servicio de Producto Sanitario. Subdirección de Análisis y Control Interno. Dirección Gerencia del Servicio Andaluz de Salud. Consejería de Salud de la Junta de Andalucía, 2010

Ruiz-Quiñonez, G.; Reza-López, S. A.; Chávez-Corral, D. V.; SánchezRamírez, B.; Leal-Berumen, I. \& Levario-Carrillo, M. Placental maturity, hypertensive disorders of pregnancy and birth weight. Hypertens. Pregnancy, 33(2):132-44, 2014.

Stoll, C.; Alembik, Y.; Dott, B. \& Roth, M. P. Study of placenta of children born with congenital malformations. Ann. Genet., 46(1):1-5, 2003.

Weida, J. N.; Schubert, F. P.; Pastrick, M. A. \& Patil, A. S. Comprehensive review of the stillborn placenta. J.Midwifery Women Health, 60(4):380-9, 2015 .

\section{Dirección para correspondencia:}

Dora Virginia Chávez Corral

Laboratorio de Embriología, Facultad de Medicina y Ciencias Biomédicas

Circuito Universitario Campus II

Universidad Autónoma de Chihuahua

C.P. 31109

Chihuahua - MÉXICO

Received: 21-07-2016

Email: dcorral@prodigy.net.mx
Accepted: 15-12-2016 